\title{
Comparative Analysis of His Bundle Versus Septal Versus Right Ventricuar Apical Pacing- A Short Term Follow up Study
}

\author{
Sudeb Mukherjee*, Asit Das and SC Mandal \\ Department of Cardiology, IPGME\&R-SSKM Hospital, India
}

*Corresponding author: Sudeb Mukherjee, Department of Cardiology, IPGME\&R-

SSKM Hospital, Kolkata, West Bengal, India.

Received Date: June 27, 2020

Published Date: July 20, 2020

\begin{abstract}
Introduction: His Bundle Pacing (HBP) is devoid of consequences of remodelling induced by Right Ventricular Apical Pacing (RVAP). Septal pacing has also been studied in several studies. Complications of heart failure, chamber dilatation and QRS prolongation are not seen in HBP and less in septal pacing. This study was done to evaluate outcomes of HBP versus Septal pacing versus RVAP in those patients requiring pacing.

Methods: Total 100 patients with atrio ventricular block with syncope who required pacing were included in this study. 50 patients underwent RVAP, 38 patients septal pacing and 12 patients underwent His bundle pacing. Detail echocardiogram-based parameters were obtained before and post pacing 6 months. All data collected appropriately and analysed accordingly using SPSS software by 1-way ANOVA with the use of the Holm-Sidak method for pairwise multiple comparisons or by the $\chi 2$ test, as appropriate. The statistical test of main treatment effect was an adjusted F test with Kenward-Roger type adjustment of denominator degrees of freedom.
\end{abstract}

Results: HBP was associated with improvements in Left Ventricular Ejection Fraction (LVEF) and other chamber dimension which was statistically significant. Septal pacing was found to be better (not statistically significant although numerically better) compared to RVAP group which showed deterioration of LVEF, chamber dimension and QRS duration.

Conclusion: HBP was found to be more physiological than septal and RVAP group although technically challenging and required expertise.

Keywords: His Bundle Pacing (HBP); Septal pacing; Right Ventricular Apical Pacing RVAP); Left Ventricular Ejection Fraction (LVEF)

\section{Introduction}

The cardiac conduction system is a collection of nodes and specialised conduction cells that initiate and co-ordinate contraction of the heart muscle. It consists of sinoatrial (SA) node, atrioventricular (AV) node, atrioventricular bundle (bundle of His) amd purkinje fibres. The sequence of electrical events during one full contraction of the heart muscle starts from SA node spreads across the atria to the AV node, into the bundle of His, down the interventricular septum and via the Purkinje fibres spread along the ventricles, causing them to contract. This rapid conduction allows coordinated ventricular contraction (ventricular systole) and blood is moved from the right and left ventricles to the pulmonary artery and aorta respectively [1-3]. Since the era of Hippocrates (460-375BC) physicians were aware of syncope. Innovative works by different scientists at different times enriched the history of pacemakers gradually. It started from McWilliam [4] to Lidwell (who developed first external pacemaker) [5] to Hyman's device (used DC current through a bipolar needle electrode) and finally to Zoll [6] who modernized the concept of pacing radically. Over the 
time through the hand of Earl E. Bakken the first battery-operated wearable pacemaker came into availability later that year for the treatment of heart bloc [7-9]. Microprocessor-driven pacemakers appeared in around 1990. These became very complex devices capable of detecting and storing events utilising several algorithms. The rate-response pattern also adjusted itself automatically to the patient's activity level.

From the first human implantation the right ventricular (RV) apical pacing has saved millions of lives, but within one decade it was proved to be non-physiological as several deleterious hemodynamic effects were noted during follow up [10]. Chronic RVAP causes left ventricular dilatation and reduction of left ventricular ejection fraction [11]. During RVAP the conduction of the electrical wave front propagates through the myocardium, rather than through the His-Purkinje conduction system as a result the electrical wave form propagates more slowly and induces heterogeneity in electrical activation of myocardium comparable to left bundle branch block [12]. Left ventricular diminished dp/dt changes (pressure changes) has also been found with chronic RVAP [13]. Decrease in contractile sate of LV myocardium leading to LV systolic dysfunction has also been noted in several studies. Subcellular anatomical changes have also been demonstrated in several previous studies that includes various histopathologic abnormalities in paced patients. Histopathological alterations in biopsy samples following pacing include myofiber variation, fibrosis, fat deposition, sclerosis, and mitochondrial morphological changes [14]. Unfavourable effects of right ventricular (RV) pacing include ventricular remodelling, dilation, elevated diastolic filling pressure, increased functional mitral regurgitation, myocardial perfusion defects, and reduced $\mathrm{LV}$ ejection fraction. Of all ventricular sites, the RV apex seems to be the most hemodynamically unfavourable [15]. Right ventricular apical pacing (RVAP) has been shown to increase morbidity and mortality in patients receiving a high percentage of cumulative pacing [16]. All these factors lead to search for alternative pacing site. Various alternative pacing sites has been explored that includes Right Ventricular Outflow Tract pacing, Septal Pacing, Biventricular Pacing, Atrial Pacing, Dual Pacing and His Bundle pacing.

\section{Septal pacing}

The idea of septal pacing is based on the fact that the septal region of the RVOT and mid RV are the first zones of the ventricle to depolarise, suggesting that pacing from these areas on the right side of the septum would achieve as normal a contraction pattern as possible.

\section{Evolution of his bundle pacing}

The term 'physiological' was used in Canadian Trial of Physiological pacing (СTOPP) to reflect the terminology at the time of development of the trial [17]. However atrial pacing was considered physiological at that time. Later it was thought that pacing the atrium and ventricle sequentially may solve the problem of unsynchronised contraction and prevent atrial bradycardia but the ventricular activation sequence is clearly not physiological. Studies using biventricular pacing have suggested improvement in patients of left bundle branch block. The dual chamber and VVI implantable defibrillator (DAVID study) randomised patients receiving implantable defibrillators (ICD) either to backup pacing at the rate of $40 \mathrm{bpm}$ or to DDDR pacing at the at the rate of 70 per minutes. The composite end point of death or hospitalisation with heart failure was greater in the group receiving DDDR pacing than in the back up pacing group [18]. There was a very similar benefit with regard to reduction of atrial fibrillation and a significant but weak benefit with regard to hospitalisation for heart failure. Two probable solutions to this problem are: first involves manipulation of pacing modes and timing cycle operation among patients with reliable atrio-ventricular conduction to minimise unnecessary ventricular pacing and preserve normal ventricular conduction and the second involves pacing at alternative ventricular site to attenuate the adverse effects imposed by ventricular desynchronization when ventricular pacing can't be avoided.

The main purpose of permanent cardiac electrostimulation is to maintain an adequate cardiac rhythm, trying to restore the physiology of the normal excito-conductive physiology of the heart as much as possible. Up until now, importance had been given to two elements that were considered fundamental for physiological pacing: maintenance of the atrioventricular Sequence and the rateresponsive function. If the aim is to mimic physiological activation patterns, the HBP may provide the ideal site. During the last several years there was much work done in the field of DHBP, to extend the indications and to improve the technique. Here in this study the comparative analysis among RVAP, His Bundle Pacing and Septal pacing has been done.

\section{Objective}

To find out outcomes of different parameters in 3 different types of pacing, His bundle vs Septal vs RV Apical in selected group of patients. This was a short term follow up study with detail analysis in respect of various parameters mentioned below.

\section{To look for ECG QRS duration shortening.}

2. To look for Left ventricular Ejection Fraction (LVEF) immediately, after 1 month and after 6 months.

3. To look for Left ventricular Internal Diameter (LVIDD) change at 1 month and at 6 months.

4. To look for Tricuspid Regurgitation (TR) in different group of pacing.

5. To assess biochemical parameters in different groups following pacing.

LV dimensions were measured from the parasternal long-axis M-mode and expressed as Z scores with the use of weight-related normal limits. LV shortening fraction was calculated. 
$\mathrm{LV}$ volumes were measured from the apical 4- and 2-chamber views with the Simpson biplane method. LV ejection fraction (EF) was calculated and graded as follows: normal

(LV EF $\geq 55 \%$ ), subnormal (LV EF $<55 \%$ ), and significantly decreased (LV EF $<45 \%$ )

\section{Methods}

All patients with atrio ventricular block presented/admitted to IPGME\&R Hospital in the department of cardiology were included in this study. It was an prospective observational study for total patients of, who were followed for 6 months and all details parameters were documented and analysed accordingly.

\section{Inclusion criterias}

For HBP: Only patients who met the following inclusion criteria were considered candidates for permanent HBP:

a) Indication of permanent pacing for $\mathrm{AV}$ conduction disturbance or left ventricular resynchronization not possible via the coronary sinus.

b) Potential for elimination of $\mathrm{AV}$ block or bundle-branch block by HBP, leading to a narrow QRS complex (120 ms).

c) Maximum hisian capture threshold of $2.5 \mathrm{~V} / 1 \mathrm{~ms}$.

d) 1:1 His-ventricular conduction at a minimum pacing rate of 120 b.p.m.

\section{For Septal Pacing}

A. Indication of permanent pacing for $\mathrm{AV}$ conduction disturbance or left ventricular resynchronization not possible via the coronary sinus and not possible to pace via his bundle.

B. Potential for elimination of AV block or bundle-branch block (LBBB) by HBP, leading to a narrow QRS complex (120 ms).

For RVAP pacing: For all other patients needed pacing included in this category.

\section{Exclusion criterias}

For HBP and Septal Pacing:

1. Complete Heart Block.

2. Wide Complex Tachycardia during any episode before and after admission.

\section{Parameters used}

Echocardiographic criterias: 1)Left Ventricular Internal Dimension in Systole and Diastole (LVIDs, LVIDd), 2)Left Ventricular Posterior Wall Thickness (LVPWD), 3)Left Ventricular Ejection Fraction (LVEF), 4) Left Atrial Diameter 5) Right Atrial Diameter 6) Right Ventricular Diameter 7) Tricuspid Regurgitation (TR).

Electrocardiographic criterias: QRS duration, QRS morphology.
Blood investigations: N-Terminal pro Brain Natriuric Peptide.

Study technique: Patients of all ages are going to be included in this study. So, after taking a valid consent from the patients, a detailed history and clinical examination was carried out.

HBP: Procedure HBP was performed using the Medtronic pacing lead delivered through a fixed curve sheath. The delivery sheath was inserted into the right ventricle beyond the tricuspid annulus over a guide wire through left cephalic or axillary vein. Subsequently, the pacing lead was advanced through the sheath such that only the distal electrode /screw is beyond the tip of the catheter. A unipolar electrogram was recorded from the lead tip at again setting of $0.05 \mathrm{mV} / \mathrm{mm}$ and displayed on a Medtronic pacing system. The preformed double curve of this catheter points the tip toward the superior AV septum. An HB electrogram was identified by mapping the AV septum. The lead was then screwed in this position by means of 4-5 clockwise rotations. The HB capture threshold was assessed and accepted if found to be $2.5 \mathrm{~V}$ at $1.0 \mathrm{~ms}$. If an acceptable HB capture could not be achieved after 5 attempts at lead positioning or a fluoroscopy duration of 30 minutes ,the lead was then placed in the RV mid-septum.

RVAP: RV leads were implanted in a standard fashion at the RV apex . Traditional tined-tip pacing to anchor a lead in the RV apex in those group of patients.

Septum: Septal pacing was done by the methods as described by Mond et, al. Under fluoroscopic imaging in the right anterior oblique view of 30 degrees, the RV was divided into 3 zones as follows: (1) an upper zone (one-third from the top of the RV) between the pulmonary artery bulge and the roof of the tricuspid valve; (2) a middle zone; and (3) a lower zone (one-third from the bottom of the RV). Then, the ventricular lead was anchored in the middle zone of the RV. After fixation of the ventricular lead, the left anterior oblique view of 45 degrees was used to confirm that the lead was successfully placed on the RV septum and not the free wall. Surface and device electrocardiography, and echocardiograpic examination studies were done by be performed at 24 hours after pacemaker implantation using a Vivid 7 Dimension ultrasound machine (GE Healthcare) with an M4S probe and again at 6 months. Echocardiographic parameters were recorded immediately after 1 months and after imlantaton and again at 6 months.

\section{Analysis of data}

All data collected appropriately and analysed accordingly. SPSS software latest version was used for demographic as well as objective analysis. ANOVA was done and analysed properly. Continuous data are presented as raw means (SDs). Differences in demographic and informative variables between pacing sites were evaluated by 1-way ANOVA with the use of the Holm-Sidak method for pairwise multiple comparisons or by the $\chi 2$ test, as appropriate. The continuous outcome variables characterizing $\mathrm{LV}$ function and synchrony were analysed with the use of a linear mixed model approach. Each model included the set of clinically 
informative additive covariates in addition to the main factor tested. The continuous covariates included age at implantation pacing duration, and QRS duration. The statistical test of main treatment

\section{Result and Analysis}

\section{Age demography}

effect was an adjusted F test with Kenward-Roger type adjustment of denominator degrees of freedom

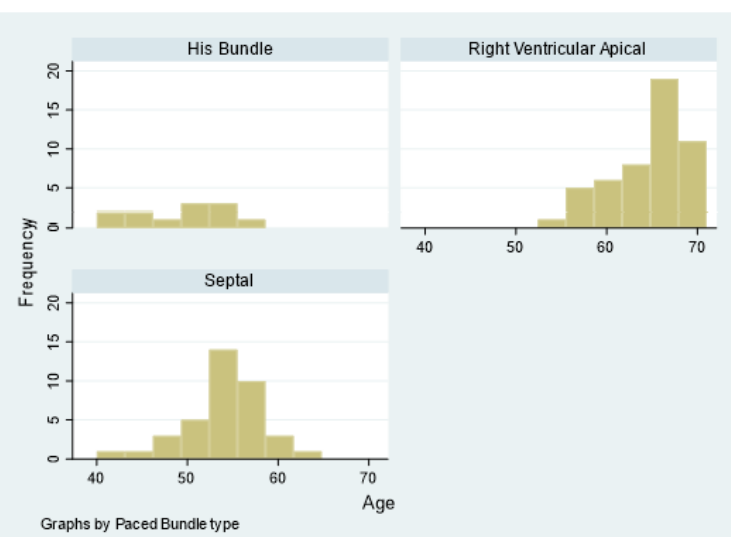

Figure 1: Showing distribution of age demography in different pacing groups.

The age of the study population in this study ranged from 40 years to 71 years. In RVAP group the age varies from 54 years to 71 years with mean of 64.24 years. In Septal pacing group the age varies from 40 years to 62 years with mean value of 53.92 years. In His bundle pacing group age varies from 43 years to 58 years with mean value of 54.45 years (Figure 1 ).

\section{Gender distribution}

The details of gender distribution has been depicted in the following chart. Out of total population 62 patients were male and 38 were female (Figure 2).

\section{Pacing distribution}

Out of total 100 patients 50 patients underwent RVAP, 38 patients septal pacing and 12 patients underwent His bundle pacing.

\section{LVEF distribution}

Mean LVEF in 3 different group has been depicted in the following chart. For RVAP group it is $62 \%$ with SD of 3.75, for Septal pacing group it is 52.95 with SD of 5.08 and for His Bundle pacing group it is $53.92 \%$ with SD of 3.77 (Figure 3).

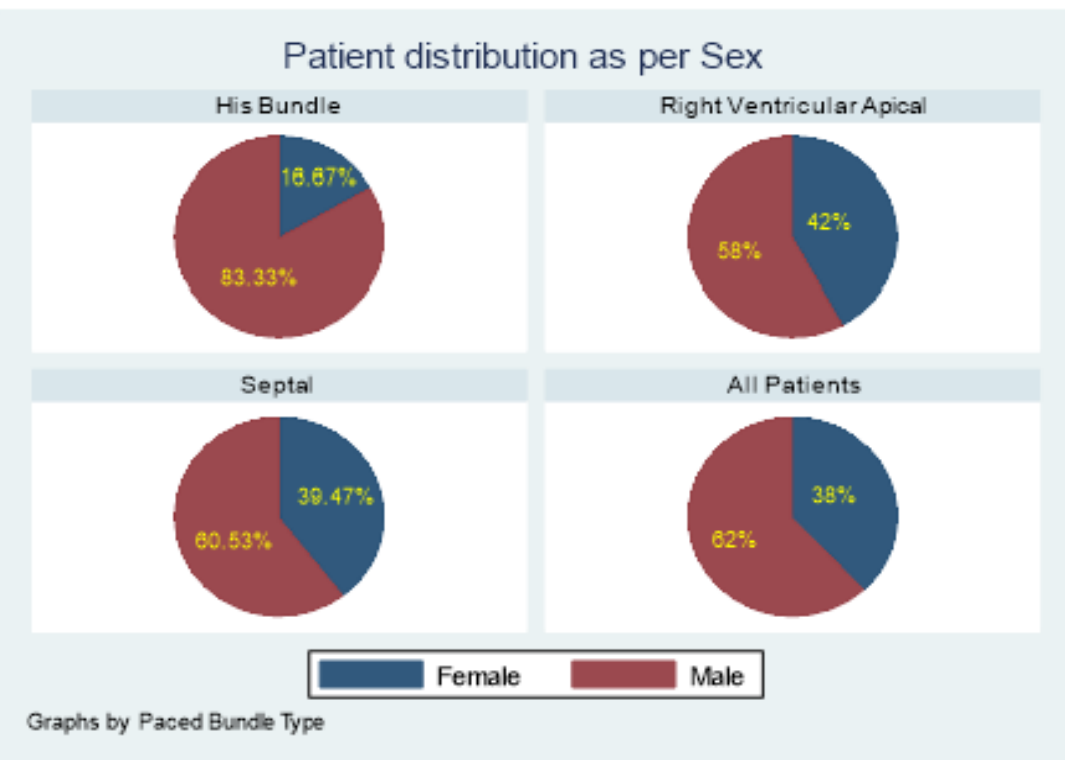

Figure 2: Showing gender distribution of study populations. 
Baseline Ejection Fraction (Mean, Standard Deviation)

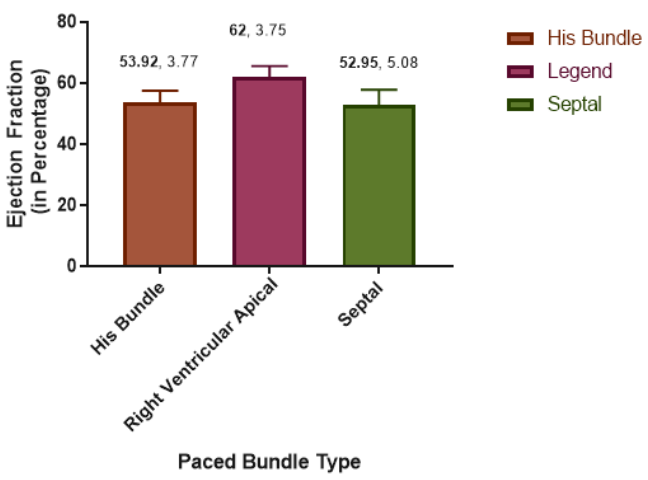

Figure 3: Distribution of baseline LVEF in different subset population.

\section{LVEF at 6-month Post Pacing}

The following chart showing changes in LVEF at 6 months following pacing. In the Septal group mean is 54.18 with SD 4.81, in the His Bundle Group it is 59.92 with SD 3.2 and in the RVAP group it is $55.14 \%$ with SD of 2.80 (Figure 4).

\section{LVEF changes pattern post pacing}

The following graph (Figure 5) shows the changes over time of LVEF of 3 groups. Of the 3 groups His bundle and septal pacing has shown improvements in LVEF whereas RVAP has been associated with decrease in LVEF.

\section{Ejection Fraction 6-month Post-Pacing (Mean, Standard Deviation)}

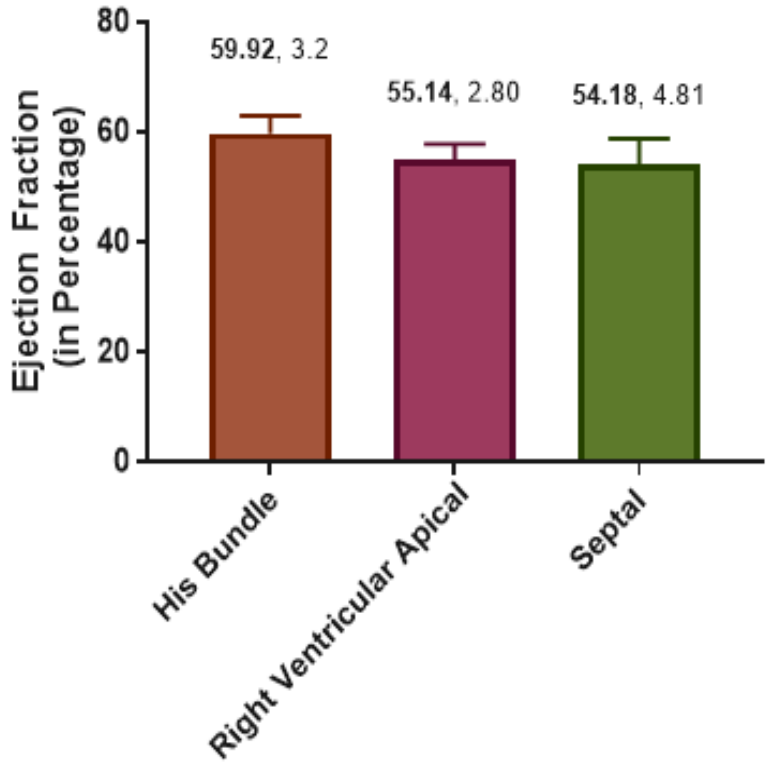

His Bundle

口 Legend

口 Septal

\section{Paced Bundle Type}

Figure 4: Showing distribution of LVEF 6 months post pacing. 


\section{Ejection Fraction in all patients grouped by bundle being paced}

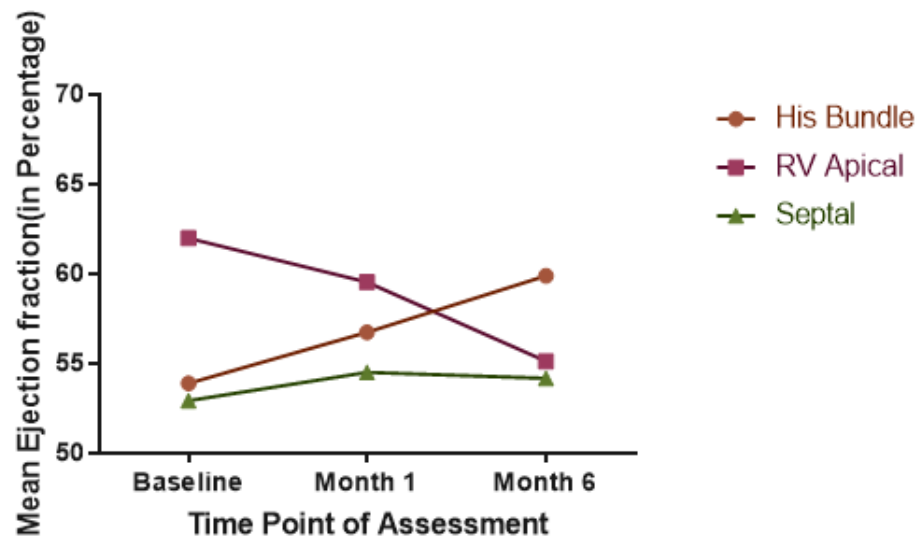

Figure 5: Showing changes in LVEF following pacing in 6 months course.

\section{LVIDD following pacing}

The following chart (Figure 6 \& 7) and graph showing distribution of baseline LVIDD in each group in the initial bar diagram. Second bar at 1 month and 3rd one showing LVIDD at 6 month following pacing in each group. The chart showing the trend of changes in LVIDD in each group over this time. For HBP group baseline, at 1 month and 6 months mean LVIDD was $46.86 \mathrm{~mm}$ (SD4.49), $48.76 \mathrm{~mm}$ (SD-4.90), $48.26 \mathrm{~mm}$ (SD 4.80) respectively. In Septal group it was $47.00 \mathrm{~mm}$ (3.81), $48.46 \mathrm{~mm}$ (SD-4.85), 61.24 $\mathrm{mm}(\mathrm{SD}-5.28)$ and in RVAP group 46.86mm (SD3.87), $48.06 \mathrm{~mm}$ (SD4.23), 61.08mm (SD-4.57) for Vaseline, 1 and 6 month respectively.

\section{Distribution of QRS duration}

Below graph is showing QRS duration change in each group following pacing compared to baseline. In the HBP group QRS dutration changed from $155.2 \mathrm{~ms}$ to $110.4 \mathrm{~ms}$. In the RVAP group it was $139.5 \mathrm{~ms}$ at baseline and 143.1 post pacing. In the Septal group it was 144.3 and 121.4 at baseline and post pacing respectively (Figure 8).

\section{NT pro BNP level following pacing}

Mean value of NT pro BNP level following pacing has been depicted in the below chart. It is very evident that NT pro BNP level has been elevated in each group (Figure 9).

\section{LVDD pre-pacing, at 1-month and 6-months post-pacing (Mean, Standard deviation)}

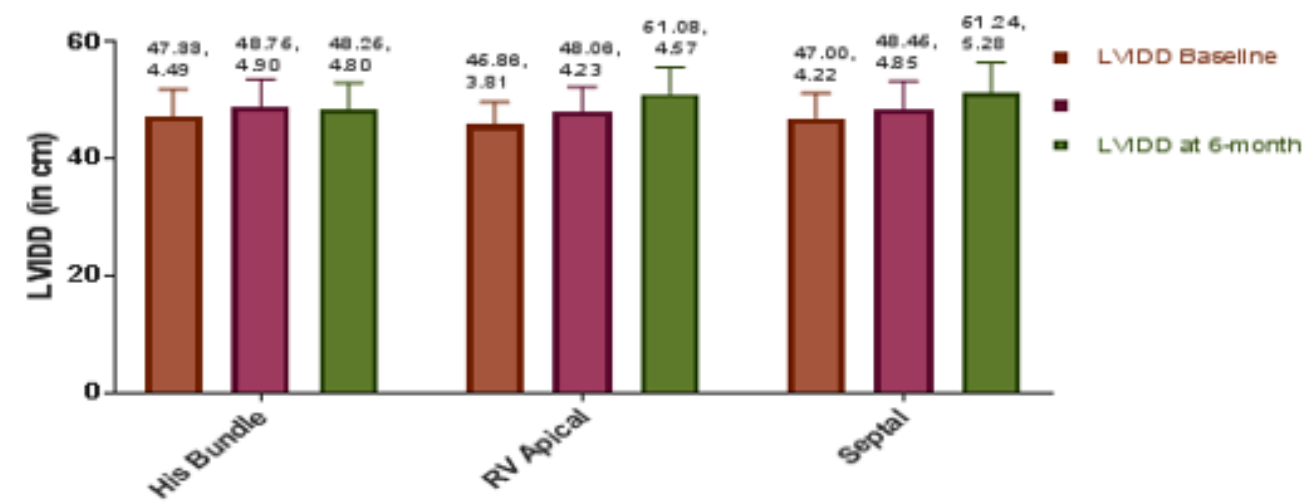

Paced Bundle Type

"LMDD: Left Ventricular Internal DI ameter in Diastole

Figure 6: Bar diagram showing distribution of LVIDD in different subsets at baseline, 1 and 6 months. 
LVIDD in all patients grouped

by bundle being paced

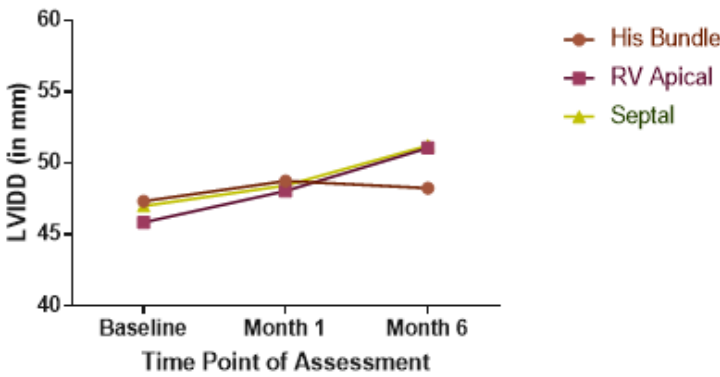

"LVIDD: Left Ventricular Internal Diameter in Diastole

Figure 7: Graph showing changes in LVIDD from baseline to post pacing at 1 and 6 months.

\section{Distribution of QRS intervals Before and After Pacing (Mean, Standard deviation)}

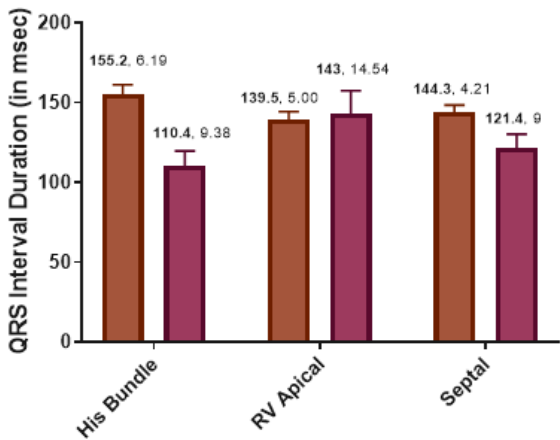

- QRS Pre-Pacing

口 QRS Post-Pacing

Paced Bundle Type

Figure 8: Showing QRS duration at baseline and post pacing different subsets of the study population.

\section{NT-proBNP levels in all patients after pacing \\ (Mean, Standard Deviation)}

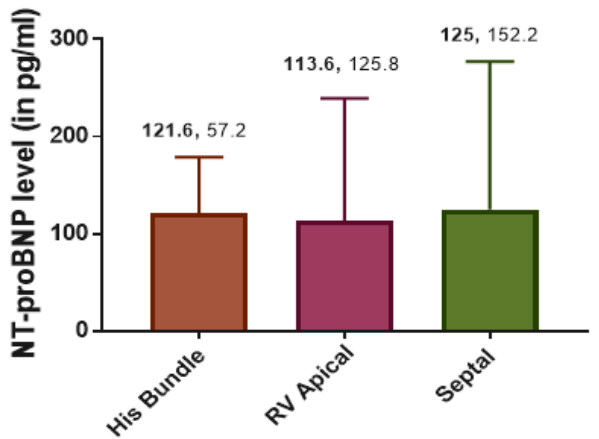

Paced bundle type

Figure 9: NT Pro BNP level in all subsets of population post pacing. 


\section{LA diameter Pre vs Post pacing}

The (Figure 10) showing baseline LA size and changes pattern and numerical value with bar diagram at 1 month and at 6 months in each group.

\section{RV diameter pattern post pacing}

The following bar diagram (Figure 11) showing baseline and post pacing RV diameter changes following pacing.

\section{Left Atrial Internal Diameter at baseline, 1-month and 6-months} post-pacing (Mean, Standard deviation)

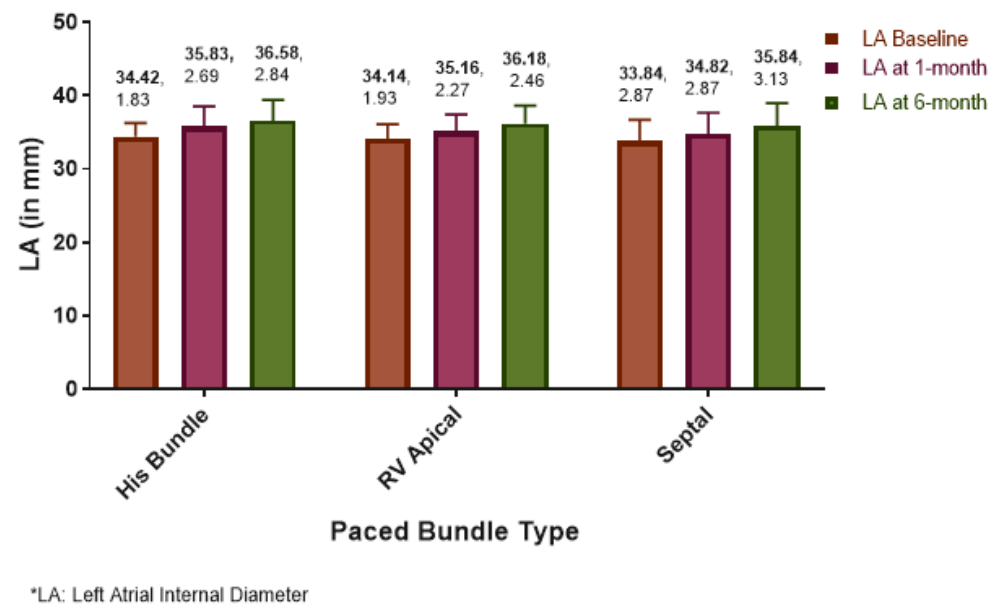

Figure 10: Bar diagram showing changes in LA diameter over time.

\section{Right Ventricular Internal Diameter at baseline, 1-month and 6-months post-pacing (Mean, Standard deviation)}

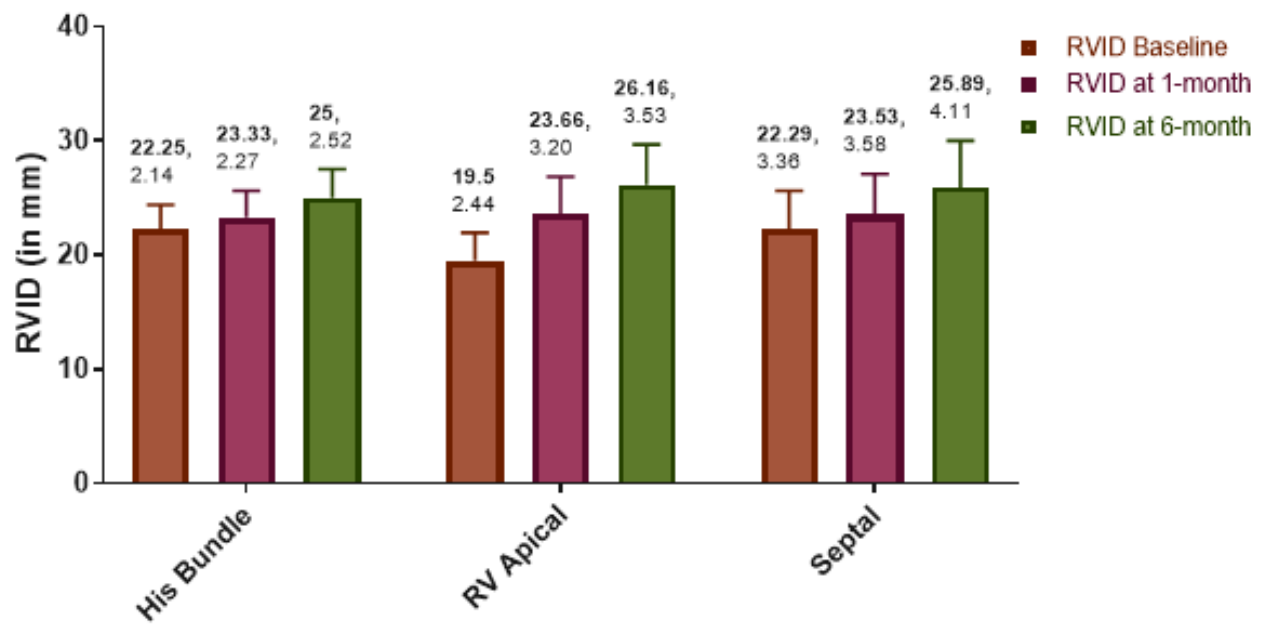

Paced Bundle Type

*RVID: Right Ventricular Internal Diameter

Figure 11: Bar diagram showing RV diameter changes over time.

\section{Tricuspid regurgitation}

The following chart (Figure 12) showing Tricuspid Regurgitation following pacing in each group. Average TR in mm of Hg for RVAP,
Septal and HBP are 23.72 (SD-7.47), 20.68(SD-9.81) and 18.08(SD3.89) respectively. 


\section{Tricuspid Gradient in all patients at baseline (Mean, Standard Deviation)}

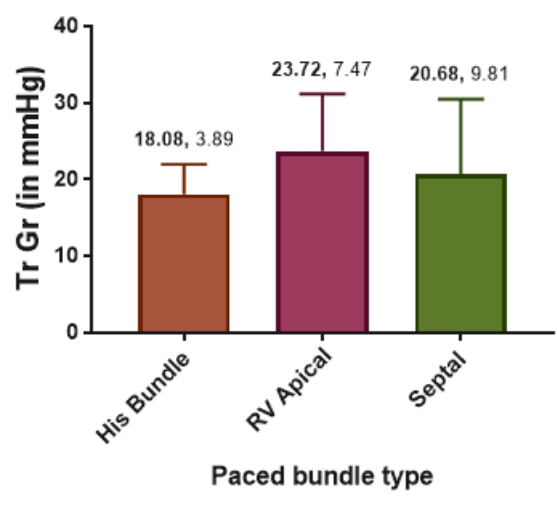

*Tr Gr: Tricuspid Gradient

Figure 12: TR gradient average in different subset of study population.

\section{Discussion}

First observation regarding HB electrophysiological characteristics was perhaps done by Scherlag in 1967 [19]. Narula in 1977 reported that, stimulation from the proximal HB showed wide QRS complexes and/or axis deviation identical to that seen during normal sinus rhythm, whereas distal HB stimulation resulted in a narrow QRS complex (95 ms) and/ or abolition of left axis deviation (LAD) [20]. In 1979 El Sheriff showed temporary HB stimulation can revert a pre-existing bundle branch block [21]. In 1999 Amitani confirmed the feasibility of clinical application of permanent HB pacing in six adult beagles in which a screw-in lead was anchored in the His-bundle region [22]. In this study we have used active fixation screwing lead in all HBP and Septal pacing patients. This study showed improvements in LVEF in HBP group which was found to be statistically significant ( $\mathrm{p}<$ .05). Mean LVEF has improved from baseline value of $53.92 \%$ to $59.92 \%$ at 6 months. Septal pacing was found to improve LVEF from baseline of $52.95 \%$ to $54.18 \%$ at 6 months. which was not statistically significant $(\mathrm{p}=0.51)$ although numerically higher value was obtained. RVAP was associated with significantly drop in LVEF. Mean baseline LVEF in RVAP group was $62 \%$ and post pacing at 6 months it was found to be 55.14\% (Figure 4). The changes in LVEF can be easily understood from (Figure 5). Deshmukh et, al. first described successful permanent His-bundle pacing (HBP) in a small series of patients with $\mathrm{AF}$ and dilated cardiomyopathy in 2000, which have demonstrated that it is feasible and associated with an improvement in exercise capacity ventricular synchrony, and LVEF as compared with RVAP. The results in our study in this short term follow up was found to be significant.

Moreover, LVIDD in this study was found to be improved in both his bundle as well as septal pacing but not with RVAP (Figure 6 \& 7). Demonstrated clearly that LVIDD increased over time in
RVAP group where as HBP and septal pacing both have not shown increased LVIDD. Finding of such parameter was new in medical literature. LA diameter was found to be increased in every varieties of pacing in this study. No significant difference was found among different types of pacing (Figure 10). RV diameter was increased from baseline in each types of pacing. Maximum dilatation was seen in RVAP group (Figure 11).TR gradient increased in all types of pacing. It was not statistically significant although (Figure 12). Maximum gradient was found in RVAP group. These findings can be corroborated with the findings from the DAVID trial (The Dual Chamber and VVI Implantable Defibrillator trial) and in the MOST trial. NT pro BNP level was found to be increased in every subsets of population be it RVAP, HBP or Septal pacing. No direct correlation was found to be significant. RVAP was found to be numerically higher value compared to rest of the two groups (Figure 9).

QRS duration changed maximum in the HBP group. In the HBP group average QRS duration changed from $155.2 \mathrm{~ms}$ at baseline to $110.4 \mathrm{~ms}$ post pacing. In the Septal group QRS duration also decreased from average of $144.3 \mathrm{~ms}$ at baseline to $121.4 \mathrm{~ms}$ post pacing. Decrease in HBP group was almost double compared to septal group. However, QRS duration increased in cases of RVAP group. In the RVAP group it was $139.5 \mathrm{~ms}$ at baseline and became 143.1 ms post pacing (Figure 8). The significant narrowing in HBP is similar to several other studies $[23,24]$. The operative success rate of HBP in several study varies from $66 \%$ to $95.5 \%$ [23-25]. In our study we did implant HB pacing successfully in all patients. Case selection, HB catheter and long sheath along with technological advancement may help in operative successfulness [26].

\section{Conclusion}

Physiological pacing 'may be achieved only by preserving, or, if that is impossible, by restoring or attempting to imitate the 
normal electrophysiological characteristics of the heart. Because His-bundle pacing (HBP) produces ventricular contraction via the specific conduction system, it does not induce interventricular or intraventricular asynchrony. It is also devoid of mechanical injury or myocardial perfusion disorders described with right ventricular apical pacing. Decrease QRS duration was found maximum in HBP group. Pacing pattern more likely to be physiological in HBP group. Some of the patient achieved close to $90 \mathrm{~ms}$ QRS duration. This narrowing may be the responsible factor for maintaining and improvements of LV function. Septal pacing has also shown better parameter compared to RVAP group in terms of RV diameter, LA diameter, degree of TR. However, HBP group superseded all and therefore should be the choice when applicable. Although HBP poses little challenge in terms of technical aspects but not very difficult now, thanks to improvements in different long sheaths and various screwing active fixation system. Thorough anatomical knowledge however is very much essential to avoid damage to atrio ventricular node during implantation.

\section{Acknowledgement}

We acknowledge contribution from all persons for giving us consent.

\section{Conflict of Interest}

No conflict of interest.

\section{References}

1. Sharma PS, Dandamudi G, Herweg B, David Wilson, Rajeev Singh, et al (2018) Permanent His-bundle pacing as an alternative to biventricular pacing for cardiac resynchronization therapy: A multicentre experience. Heart Rhythm 15: 413-420.

2. Cho GY, Kim MJ, Park JH, Kim HS, Youn HJ, et al. (2011) Comparison of ventricular desynchrony according to the position of right ventricular pacing electrode: A multi-center prospective echocardiographic study. J Cardiovasc Ultrasound 19: 15-20.

3. Shukla HH, Hellkamp AS, James EA, Flaker G, Lee K, et al. (2005) Heart failure hospitalization is more common in pacemaker patients with sinus node dysfunc-tion and a prolonged paced QRS duration. Heart Rhythm 2: 245-251.

4. McWilliam JA (1889) Electrical stimulation of the heart in man. Br Med J 1(1468): 348-350.

5. Lidwell MC (1929) Cardiac disease and anaesthesia, Austral Asian medical congress (British medical association). Australia Med J Austral 2: $574-575$.

6. Zoll PM (1952) Resuscitation of the heart in ventricular standstill by external electric stimulation. N Engl J Med 247(20): 768-771.

7. Lee MA, Dae MW, Langberg JL, Griffin JC, Chin MC, (1994) Effects of long-term right ventricular apical pacing on left ventricular perfusion, innervation, function, and histology. J Am Coll Cardiol 24: 225-232.

8. Karpawich PP, Rabah R, Haas JE (1999) Altered cardiac histology following apical right ventricular pacing in patients with congenital atrioventricular block. Pacing Clin Electrophysiol 22: 1372-1377.

9. Lindsay BD (2009) Deleterious effects of right ventricular pacing. N Engl J Med 361: 2183-2185.
10. Mukherjee S (2018) His Bundle Pacing: Short Review of Literature and Technical Aspects. J Clin Exp Res Cardiol 4(2): 201.

11. Prinzen FW, Augustijn CH, Arts T, Allessie MA, Reneman RS (1990) Redistribution of myocardial fiber strain and blood flow by asynchronous activation. Am J Physiol 259: H300-8.

12. Connolly SJ, Kerr CR, Gent M, Roberts RS, Yusuf S, et al. (2000) Effects of physiologic pacing versus ventricular pacing on the risk of stroke and death due to cardiovascular causes. Canadian Trial of Physiologic Pacing Investigators. N Engl J Med 342: 1385-1391.

13. Karpawich P, Mital S (1997) Comparative left ventricular function following atrial, septal, and apical single chamber heart pacing in the young. Pacing Clin Electrophysiol 20: 1983-1988.

14. Cicchitti V, Radico F, Bianco F, Gallina S, Tonti G, et al. (2016) Heart failure due to right ventricular apical pacing: the importance of flow patterns. Europace 18(11): 1679-1688.

15. Gold MR, Brockman R, Peters RW, Olsovsky MR, Shorofsky SR (2000) Acute hemodynamic effects of right ventricular pacing site and pacing mode in patients with congestive heart failure secondary to either ischemic or idiopathic dilated cardiomyopathy. Am J Cardiol 85: 11061109.

16. Tse H-F, Lau C-P (1997) Long-term effect of right ventricular pacing on myocardial perfusion and function. J Am Coll Cardiol 29: 744-749.

17. Mond HG, Hillock RJ, Stevenson IH, McGavigan AD (2007) The right ventricular outflow tract: the road to septal pacing. Pacing Clin Electrophysiol 30(4): 482-491.

18. Wilkoff BL, Cook JR, Epstein AE, Greene HL, Hallstrom AP, et al. (2002) Dual-chamber pacing or ventricular backup pacing in patients with an implantable defibril-lator: The Dual Chamber and VVI Implantable Defibrillator (DAVID) Trial. JAMA 288: 3115-3123.

19. Nielsen JC, Kristensen L, Andersen HR, Mortensen PT, Pedersen OL, et al. (2003) A randomized comparison of atrial and dual-chamber pacing in 177 consecu-tive patients with sick sinus syndrome: echocardiographic and clinical outcome. J Am Coll Cardiol 42: 614-623.

20. Scherlag BJ, Helfant RH, Damato AN (1968) A catheter technique for His bundle stimulation and recording in the intact dog. J Appl Physiol 25: 425-428.

21. Narula OS, Scherlag BJ, Samet R (1970) Pervenous pacing of the specialized conducting system in man: His bundle and $A-V$ nodal stimulation. Circulation 41: 77-87.

22. El-Sherif N, Amay-Y-Leon F, Schonfield C, BJ Scherlag, K Rosen, et al. (1978) Normalization of bundle branch block patterns by distal His bundle pacing. Clinical and experimental evidence of longitudinal dissociation in the pathologic His bundle. Circulation 57: 473-483.

23. Amitani S, Miyahara K, Sohara H, H Kakura, M Koga, et al. (1999) Experimental his-bundle pacing: histopathological and electrophysiological examination. Pacing Clin Electrophysiol 22: 562 566.

24. Deshmukh P, Casavant DA, Romanyshyn M, Anderson K (2000) Permanent, direct His-bundle pacing: a novel approach to cardiac pacing in patients with normal His-Purkinje activation. Circulation 101(8): 869-877.

25. Barba-Pichardo R, Moriña-Vázquez P, Fernández-Gómez JM, VenegasGamero J, Herrera-Carranza M (2010) Permanent His-bundle pacing: Seeking physiological ventricular pacing. Europace 12(4): 527-533.

26. Zanon F, Baracca E, Aggio S, Pastore G, Boaretto G, et al. (2006) A feasible approach for direct His-bundle pacing using a new steerable catheter to facilitate precise lead placement. J Cardiovasc Electrophysiol 17: 29-33. 